\title{
ANÁLISIS DE LA PROBABILIDAD DE QUIEBRA DE LAS EMPRESAS COTIZADAS ESPAÑOLAS.
}

\section{ANALYSIS OF THE PROBABILITY OF BANKRUPTCY OF LISTED SPANISH COMPANIES.}

\author{
Moreno, Elena (Universidad de Sevilla) * \\ Bravo, Francisco (Universidad de Sevilla) ${ }^{* *}$
}

\begin{abstract}
Resumen:
El presente trabajo analiza diferentes cuestiones relacionadas con la probabilidad de quiebra de las empresas españolas cotizadas. En primer lugar, se ha analizado el riesgo de quiebra de estas empresas en el año 2015 en base al índice Z-Score diseñado por Altman (1968). En segundo lugar, se realiza una comparación de los resultados procedentes del cálculo del indicador Z-score con los resultados obtenidos al utilizar un indicador de la probabilidad de quiebra adaptado para las empresas españolas. Finalmente, se analiza la posible relación existente entre la probabilidad de quiebra y la calidad de la información voluntaria publicada de las empresas españolas, cuestión importante para mejorar la eficiencia de los mercados de capitales. La evidencia encontrada puede tener implicaciones directas tanto para empresas como para reguladores.
\end{abstract}

Palabras claves: Probabilidad de quiebra; Información voluntaria; Z-score; Mercados de capitales.

\begin{abstract}
:
This paper analyzes different issues related to the probability of bankruptcy of Spanish listed companies. In the first place, the bankruptcy risk of these companies in 2015 was analyzed based on the Z-Score index designed by Altman (1968). Secondly, a comparison is made of the results from the calculation of the Z-score indicator with the results obtained by using an indicator of bankruptcy probability adapted for Spanish companies. Finally, the possible relationship between the probability of bankruptcy and the quality of voluntary information published by Spanish companies is analyzed, an important issue to improve the efficiency of capital markets. The evidence found may have direct implications for both companies and regulators.
\end{abstract}

Key words: Probability of bankruptcy; Voluntary information; Z-score; Capital markets.

Clasificación JEL: G33 Quiebra

JEL classification: G33 bankruptcy

\footnotetext{
* Departamento de Contabilidad y Economía Financiera, Facultad de Ciencias Económicas y Empresariales, Avda. Ramón y Cajal 1, 41018 Sevilla. elenamoreno103@hotmail.com.

** Departamento de Contabilidad y Economía Financiera, Facultad de Ciencias Económicas y Empresariales, Avda. Ramón y Cajal, 1.| 41018 Sevilla. pacobravo@us.es.

Recibido: 12 de noviembre de 2018. Aceptado: 7 de diciembre de 2018.
} 


\section{INTRODUCCIÓN}

Las empresas españolas han sufrido de forma importante las consecuencias negativas del largo periodo de crisis financiera acontecido en los últimos años. El estudio del riesgo de quiebra es una cuestión relevante en el análisis financiero con importantes implicaciones para empresas y reguladores. En este sentido, numerosos trabajos han examinado recientemente los determinantes y las consecuencias de tener un riesgo de quiebra elevado (Manzaneque et al., 2016; Udin et al., 2017; Habib et al., 2018).

Este trabajo tiene como primer objetivo realizar un análisis de la probabilidad de quiebra de las empresas cotizadas españolas en el año 2015 en base al modelo de Altman (1968), que ha sido utilizado comúnmente en la literatura existente (Shahwan, 2015; Udin et al., 2017). Con esto se pretende estudiar la situación financiera de las empresas más representativas en España tras un largo periodo de crisis financiera, examinando los factores determinantes de dicha situación. Además, se lleva a cabo un análisis comparativo de los resultados aplicando la fórmula de Altman (1968) con los obtenidos mediante un indicador adaptado a empresas españolas (Amat, 1990). Adicionalmente, se estudia la relación existente entre la calidad de la información voluntaria suministrada por las empresas y su probabilidad de quiebra. La información divulgada por las compañías adquiere un papel importante como mecanismo para reducir las asimetrías informativas y mejorar así el funcionamiento de los mercados de capitales (Healy y Palepu, 2001; Lambert et al., 2012). Sin embargo, pocos estudios han analizado dicha relación (Holder-Webb y Cohen, 2007).

La muestra utilizada se compone de las empresas cotizadas en el Índice General de la Bolsa de Madrid (IGBM) en el año 2015, que coincide con el final de la última crisis económico-financiera sufrida en España. Los resultados ponen de manifiesto que la mayoría de empresas tenían una alta probabilidad de quiebra considerando los valores del indicador propuesto por Altman (1968). Sin embargo, los valores del indicador propuesto por Amat (1990) sugieren que las empresas con alta probabilidad de quiebra son una minoría. El análisis posterior, en 2018, de las empresas estudiadas, confirma que la gran mayoría de ellas mantiene su actividad, y por lo tanto los indicadores de quiebra, principalmente el de Altman (1968), no parecen tener una alta capacidad predictiva, al menos en el escenario analizado. Nuestro trabajo por tanto supone una contribución a la literatura existente sobre la predicción de quiebra, especialmente en el contexto español. Adicionalmente, hemos encontrado evidencia que apunta a que son las empresas con mayor probabilidad de quiebra las que publican una información de mayor calidad. Estas empresas probablemente buscan reducir problemas de agencia llevando a cabo mejores prácticas de divulgación de información voluntaria para evitar así asimetrías informativas y mejorar su situación financiera. En este sentido, este trabajo contribuye también a la literatura sobre divulgación de información, incidiendo directamente en los determinantes de la publicación de información voluntaria y en las teorías sobre divulgación de información.

El resto del trabajo se estructura de la siguiente forma. El segundo apartado incluye una revisión de la literatura. El tercer apartado hace referencia a la metodología. Los apartados cuarto y quinto presentan los principales resultados obtenidos y las conclusiones más relevantes, respectivamente. 


\section{LITERATURA PREVIA}

\subsection{La quiebra: concepto y predicción}

Se entiende por quiebra la situación jurídica en la que una persona, una empresa o una institución no puede hacer frente a los pagos que debe hacer a sus acreedores, pues estos son más elevados que los recursos económicos que posee (activos). En España, tras la Ley 22/2003 Concursal, la quiebra recibe el nombre de concurso de acreedores. El presupuesto objetivo del concurso de acreedores es la insolvencia del deudor, caracterizada por el cese generalizado de los pagos corrientes (no es necesario que exista déficit patrimonial).

Dada la importancia de esta cuestión, los diferentes grupos de interés o stakeholders demandan información al respecto para proteger sus intereses y poder tomar decisiones eficientes (Habib et al., 2018). En este sentido, existen diversos indicadores o modelos de predicción de quiebra, que pretenden distinguir las características financieras de las empresas que llegan a la quiebra de aquellas que no lo hacen. Su objetivo principal es predecir qué empresas van a quebrar en un plazo corto de tiempo (uno o dos años) antes de que ocurra este hecho, de modo que las mismas puedan cambiar la forma de gestión, reestructurarse y sobrevivir.

Podemos clasificar estos modelos que diferencian las empresas sanas de las quebradas en dos tipos (Mora, 1994): univariantes y multivariantes. Dentro de los modelos univariantes destaca el propuesto por Beaver (1966), en el que después de estudiar numerosos ratios, concluye que seis de ellos tienen un alto poder predictivo de forma individual de la probabilidad de quiebra de una empresa. ${ }^{1}$ El análisis multivariante incluye varias variables simultáneamente, y el modelo más importante de este tipo es el propuesto por Altman (1968).

El indicador Z-score construido por Altman (1968) es una herramienta que ayuda a predecir la probabilidad de quiebra. El modelo de Altman (1968) es el precursor de los modelos de análisis discriminante múltiple. Desde el modelo inicial hasta el que se usa actualmente, se han introducido varias modificaciones, Altman editó una serie de 20 artículos que intentaron perfeccionar el modelo para medir el riesgo de las compañías, (Company and Country Risk Models) aunque la metodología actual es parecida a la original. El modelo propuesto por Altman (1968) se basa en información contable y financiera, incluyendo también como un factor importante el valor de mercado de las empresas. El modelo establece unos umbrales que permiten establecer categorías para las empresas con alta probabilidad de quiebra y con baja probabilidad de quiebra. Aunque el establecimiento de umbrales implica la existencia de un margen de error (Ibarra, 2001), el modelo permite diferenciar de forma sencilla las empresas que se encuentran en zonas de riesgo de aquellas que no. Dicho modelo es una referencia en la literatura existente (Mohammed y Kim-Soon, 2012; Habib et al., 2018) sobre predicción de quiebra y ha sido utilizado ampliamente desde su aparición hasta la actualidad en investigaciones acerca de los determinantes y consecuencias de la quiebra empresarial. Así, en los últimos años, existen estudios que utilizan el indicador Z-score (Altman, 1968) para analizar la relación existente entre las opciones de crecimiento y la probabilidad de quiebra de las empresas (Charitou et al., 2011). Otros estudios usan dicho indicador para examinar cómo la tipología de inversores de una empresa puede condicionar su probabilidad de quiebra (Tykvová y Borell, 2012). El modelo de Altman (1968) ha sido también considerado en investigaciones que se han centrado en la asociación existente entre la probabilidad de quiebra y otras cuestiones, como la evasión fiscal (Richardson et al., 2015), la rentabilidad de las acciones y los flujos de efectivo de explotación (Lee et al., 2017), e incluso

\footnotetext{
${ }^{1}$ Para más información ver Beaver (1966).
} 
las relaciones con los empleados (Kane et al., 2005). Muy recientemente se han publicado trabajos que, usando el indicador propuesto por Altman (1968), proponen que la probabilidad de quiebra está relacionada con ciertos mecanismos de gobierno corporativo de las empresas (Shahwan, 2015; Udin et al., 2017) y con sus estrategias de responsabilidad social corporativa (Al-Hadi et al., 2017).

Por otra parte, el modelo ha sido revisado y adaptado en diversas ocasiones, teniendo en cuenta condiciones específicas de determinados contextos y economías. Estas actualizaciones han sido realizadas tanto por el propio autor como por otros investigadores. Por ejemplo, el modelo fue adaptado expresamente para empresas no cotizadas (Altman, 1983). Durante los años siguientes los parámetros y coeficientes fueron adaptados para diferentes situaciones (Altman et al, 1995 y Altman y Hotchkiss, 2006). El indicador Altman et al. (1995) se extendió a las empresas localizadas en países en desarrollo, y en la fórmula de predicción de quiebra se excluyó la ratio relativa a la rotación de los activos para eliminar la posible distorsión de los resultados provocada por el sector y país. El modelo posterior (Altman y Hotchkiss, 2006) se modificó para ajustar los resultados obtenidos a diferentes sectores, y consideró otras cuestiones como la exposición al riesgo de tipo de cambio y la posición competitiva de la empresa. Asimismo, el modelo ha sido adaptado por otros autores para economías concretas. En el ámbito latinoamericano, se ha empleado el modelo original ajustado a las características específicas de países como Costa Rica (Vargas, 2013) o Colombia (Berrío y Cabeza, 2015). En Europa se ha aplicado el modelo a las empresas italianas (Altman et al., 2015; Celli, 2015) y a las griegas (Gerantonis et al., 2009), mientras que en Asia ha sido empleado a las compañías chinas (Thomas et al., 2011) entre otras.

De esta forma, dado que la predicción de la quiebra puede verse condicionada por factores inherentes a los países donde las empresas desarrollan su actividad, existen también modelos diseñados a nivel español. En este sentido, en España, Amat (1990) adaptó el modelo de Altman a las empresas españolas, realizando ajustes en las variables a incluir y matizando sus ponderaciones. Amat (1990) desarrolló un estudio a nivel nacional con más de 80.000 empresas en el que detectó una serie de ratios con alta capacidad predictiva de la situación de quiebra empresarial, creando a partir de ahí un indicador que se ha convertido en referencia en el contexto español (Galindo, 2000; Llenas, J., 2006; Rodríguez y Gómez, 2016).

\subsection{La probabilidad de quiebra y la divulgación de información voluntaria}

Los determinantes y consecuencias de la probabilidad de quiebra han sido ampliamente estudiados en la literatura previa. Habib et al., (2018) clasifica las consecuencias que puede tener la situación de probabilidad de quiebra de una empresa en cuatro categorías: 1) información financiera y proceso de auditoría; 2) efectos a nivel de operaciones organizacionales; 3) consecuencias en los mercados de capitales; 4) impacto en los mecanismos de gobierno corporativo. En esta línea, la evidencia empírica sugiere que las empresas con mayor probabilidad de quiebra podrían publicar información financiera de menor calidad (Chen et al., 2010; Jacoby et al., 2016).

Sin embargo, no existe evidencia concluyente acerca de los efectos que una situación de alta probabilidad de quiebra podría tener sobre las prácticas de divulgación de información voluntaria de las empresas. Este es un tema importante debido a que tradicionalmente la divulgación de información voluntaria ha sido considerada como un mecanismo fundamental para mejorar las decisiones de los diferentes grupos de interés y la eficiencia en los mercados de capitales (Healy y Palepu, 2001). La calidad de la información es un concepto ligado a la transparencia empresarial, que es una forma de obtener la confianza de todos aquellos que se relacionan con la organización. En este sentido, diversas investigaciones empíricas han 
defendido que la información voluntaria juega un papel relevante en la generación de valor, de reputación corporativa o en la reducción del coste de financiación (Cabrera, 2008; Francis et al., 2008; Sarkar y Bhattacharjee, 2017). Por otra parte, en los últimos años, a raíz de los numerosos escándalos financieros ocurridos a principios de siglo, se han incrementado de forma notable las demandas sociales para mejorar las prácticas de divulgación de información voluntaria de las empresas.

Muchos de los estudios que han analizado la posible relación entre las características empresariales y la calidad de la información voluntaria divulgada por las empresas han fundamentado sus argumentos en la teoría de la agencia (Jensen y Meckling, 1976). Esta teoría sugiere que existen conflictos de interés entre empresas e inversores cuando se presentan asimetrías de información, y que unas prácticas de divulgación de información de mayor calidad favorecen la reducción de dichos problemas. En nuestro caso, es posible pensar que las empresas con mayor probabilidad de quiebra tengan un mayor endeudamiento y por tanto unas asimetrías informativas más notables. Por ello, con el propósito de satisfacer las necesidades de información de sus acreedores, estas empresas pueden establecer prácticas de divulgación de información voluntaria de mayor calidad (García-Meca et al., 2005).

\section{METODOLOGÍA}

\subsection{Muestra y datos}

La muestra está compuesta por las empresas que forman parte del Índice General de la Bolsa de Madrid en el año 2015, divididas en seis sectores de actividad según la clasificación del citado índice: Materiales Básicos e Industria, Servicios de Consumo Bienes de Consumo Tecnología y Telecomunicaciones, Servicios Financieros e Inmobiliarias y Petróleo y Energía. Las empresas del sector financiero han sido excluidas debido a sus particularidades en la presentación de la información contable, por lo que la muestra final la componen 87 empresas.

La elección de este año tiene un doble objetivo. Por un lado, 2015 supone el fin de una fuerte crisis económica y financiera en España, según datos macroeconómicos proporcionados por el Instituto Estadístico de España (INE), y los resultados permiten conocer la situación de las empresas al término de esta etapa. Por otro lado, los resultados contribuyen a confirmar la capacidad predictiva de los indicadores de probabilidad de quiebra, pues éstos son más efectivos teóricamente en los años sucesivos a su cálculo. Así, por ejemplo, la precisión esperada de la fórmula Altman Z-score para un año antes de la fecha de la quiebra es un 95 por cien, y para dos años antes de que la empresa llegue a la quiebra la fórmula aporta un 83 por cien de exactitud.

Los datos financieros necesarios para calcular la probabilidad de quiebra de las empresas se han obtenido de la base de datos Sistema de Análisis de Balances Ibéricos (SABI). En el caso de no estar disponibles, se usaron alternativamente como fuentes de información las cuentas anuales publicadas de cada empresa. Por otra parte, los datos relativos a la calidad de la información voluntaria divulgada por las empresas se extraen del Informe Reporta 2016, que cuantifica la calidad de la información de las empresas del IGBM en 2015 en base a tres principios: transparencia, relevancia y accesibilidad.

\subsection{Variables}

Para llevar a cabo este trabajo se han utilizado principalmente tres variables, que son explicadas a continuación: indicador Z-score de Altman (1968), indicador de probabilidad de quiebra de Amat (1990), calidad de la información voluntaria de las empresas. 
En primer lugar, el modelo de Altman actual para empresas cotizadas sugiere que el riesgo de quiebra depende de la liquidez, rentabilidad acumulada, rentabilidad, estructura financiera y rotación de activos, y el indicador Z-score se calcula de la siguiente manera:

$\mathrm{Z}$-score $=1.2 *(\mathrm{FM} / \mathrm{AT})+1.4 *($ Rdo. no distrib./AT $)+3.3 *(\mathrm{BAIT} / \mathrm{AT})+$ 0.6*(Capitalización./PT) + 1* (Cifra Negocios /AT)

Dónde: FM: Fondo de Maniobra; AT: Activo total; Rdo. no distrib.: Resultados no distribuidos, es decir, resultado del ejercicio - dividendos; BAIT: Beneficio antes de interés e impuestos; PT: Pasivo total

Cada componente de Z-score ofrece una información financiera diferente:

- FM/AT: indica si la empresa se encuentra o no en equilibrio financiero.

- Resultados no distribuidos/AT: da información sobre la política de autofinanciación de la compañía.

- BAIT/AT: muestra la rentabilidad económica, es decir, la rentabilidad de los activos de la empresa.

- Capitalización/PT: hace referencia a la valoración de mercado.

- Cifra de negocios/AT: advierte de la rotación de los activos.

La probabilidad de quiebra de una empresa dependerá del resultado de la fórmula Altman Z-score. Según el resultado, la empresa podrá encontrarse en la zona segura, zona gris o en la zona de peligro.

- $\mathrm{Z}<1.81 \rightarrow$ existe una alta probabilidad de quiebra.

- $3>\mathrm{Z}>1.81 \rightarrow$ zona gris.

- $\quad \mathrm{Z}>3 \rightarrow$ existe una baja probabilidad de quiebra.

En segundo lugar, el indicador Z adaptado para las empresas españolas (Amat, 1990) se calcula exclusivamente considerando información contable, de la siguiente manera:

$\mathrm{Z}=-4.2+1.35 *$ (Activo corriente / Pasivo corriente) $+6.5 *$ (Fondos propios / Pasivo $)+$ 7* (Beneficio neto / Activo) $+5 *$ (Beneficio neto / Capitales propios))

Cada componente ofrece la siguiente información financiera:

- Activo corriente / Pasivo corriente: hace referencia a la liquidez en base a un análisis fondo, es decir, capacidad de hacer frente a las obligaciones a corto plazo.

- Fondos propios / Pasivo: muestra la independencia financiera, la autonomía de la empresa respecto a terceros.

- Beneficio neto / Activo: advierte de la rentabilidad de los activos de la empresa.

- Beneficio neto / Capitales propios: mide la rentabilidad financiera.

Por lo tanto, los dos primeros ratios están muy relacionados con la estructura de la deuda y los dos últimos con capacidad económica o rentabilidad de las empresas. En cuanto a la interpretación, se entiende que:

- $\quad$ Si Z $>0 \rightarrow$ la viabilidad de la empresa no se ve comprometida

- Si $\mathrm{Z}<0 \rightarrow$ la viabilidad de la empresa está comprometida

Una de las principales diferencias entre ambos indicadores reside en la valoración de mercado, que sólo es considerada por el indicador Z-score propuesto por Altman (1968). Con respecto a las principales diferencias en las variables contables cabe destacar que Z-score de Altman (1968) se basa en la rentabilidad económica, mientras que el indicador adaptado para las empresas españolas depende tanto de la rentabilidad de los activos como de la rentabilidad 
financiera. Asimismo, el riesgo de quiebra medido por Z-score (Altman, 1968) se ve influenciado por la política de autofinanciación de la empresa, mientras que el indicador adaptado al contexto español está condicionado por la autonomía de la empresa frente a terceros, es decir, por su independencia financiera.

Finalmente, en España se elabora desde hace años un estudio sobre la calidad de la información, principalmente voluntaria, publicada por las empresas que integran el IGBM para evaluar la calidad de esta información. Este estudio, publicado anualmente por la empresa Deva Comunicación Financiera, recibe el nombre de Informe Reporta2, se realiza por un equipo de analistas expertos en la materia y sigue un riguroso proceso de elaboración y supervisión para garantizar la independencia y objetividad. En dicho informe se mide la calidad de la información voluntaria en base a 34 indicadores que consideran la transparencia, la relevancia, y la accesibilidad de esta información. Dichos indicadores se diseñan tomando como referencia las directrices y recomendaciones de The Global Reporting Initiative (GRI), AA1000 de Accountability, Dow Jones Sustainability Index (DJSI), FTSE4Good, y el International Integrated Reporting Council (IIRC), entre otros.

\section{ANÁLISIS}

Los resultados de este trabajo se presentan en tres apartados, que se corresponden con cada uno de los objetivos del trabajo: 1) análisis de la probabilidad de quiebra en base al modelo de Altman (1968); 2) comparar los resultados obtenidos con los procedentes del cálculo del indicador adaptado a empresas españolas (Amat, 1990); 3) estudiar la relación existente entre la calidad de la información voluntaria suministrada por las empresas y su probabilidad de quiebra.

\subsection{Análisis de la probabilidad de quiebra en base al modelo de Altman (1968)}

El cuadro 1 presenta la información detallada de los valores descriptivos del Z-score propuesto por Altman (1968), mostrando también cada uno de los ratios que lo integran.

\section{CUADRO 1. VALORES DESCRIPTIVOS DEL INDICADOR Z-SCORE DE}

ALTMAN (1968

\begin{tabular}{|l|l|l|l|l|l|}
\hline & Media & $\begin{array}{l}\text { Desviación } \\
\text { estándar }\end{array}$ & $\begin{array}{l}\text { Percentil } \\
\mathbf{2 5}\end{array}$ & Mediana & $\begin{array}{l}\text { Percentil } \\
\mathbf{7 5}\end{array}$ \\
\hline Z-score Altman & 24,78 & 102,96 & 0,65 & 1,15 & 4,39 \\
\hline $1,2 *(\mathrm{FM} / \mathrm{AT})$ & 0,04 & 0,25 & $-0,09$ & 0,04 & 0,17 \\
\hline $\begin{array}{l}1,4^{*}(\mathrm{Rdo} \text { no } \\
\text { distribuido/AT) }\end{array}$ & 0,03 & 0,19 & $-0,02$ & 0,02 & 0,08 \\
\hline $3,3^{*}($ BAIT/AT) & 0,10 & 0,37 & $-0,01$ & 0,10 & 0,23 \\
\hline $0,6 *($ Capitaliz./ PT) & 24,18 & 103,01 & 0,26 & 0,74 & 3,05 \\
\hline $\begin{array}{l}1 *(\text { Importe Neto } \\
\text { C.Negocios/ AT) }\end{array}$ & 0,43 & 0,64 & 0,05 & 0,24 & 0,56 \\
\hline
\end{tabular}

Fuente: Elaboración propia

\footnotetext{
2 Para información detallada ver informereporta.com
} 
Cabe destacar que por término medio las empresas objeto de estudio que integran el Índice General de la Bolsa de Madrid tienen una baja probabilidad de quiebra (Z-score 24,78, muy superior a 3). En promedio, todos los componentes del indicador Z-score ponderados son positivos, destacando la (Capitalización/ PT), que es la ratio que explica en mayor proporción el comportamiento del indicador. Sin embargo, los datos muestran una dispersión muy alta en dicha ratio y, en consecuencia, en el indicador Z-score. El valor de la mediana es de 1,15, inferior a 1,81, lo que manifiesta que existen muchas empresas, al menos la mitad, que se encuentran en una alta probabilidad de quiebra. Por lo tanto, el valor promedio de Z-score está muy sesgado al alza por los altos valores de capitalización de un número reducido de empresas.

Un análisis pormenorizado de cada uno de los ratios permite sacar otras conclusiones relevantes:

- En el contexto analizado los datos contables de las empresas que componen la muestra son muy desfavorables, lo que demuestra el efecto negativo de la crisis sobre las cuentas anuales de estas compañías. Las empresas que se encuentran en situación de baja probabilidad de quiebra no lo están por sus datos contables, sino por sus valores de mercado. En un contexto de crisis, la capitalización juega por lo tanto un papel determinante en la interpretación del indicador Z-score de Altman (1968).

- La otra ratio que parece contribuir en mayor medida a explicar los valores del indicador Z-score es el relativo a la rotación de activos (cifra de negocios dividido por activo total). Las empresas, a pesar de las consecuencias que la crisis pudo tener en la facturación mantienen rotaciones aceptables probablemente por el ajuste llevado a cabo en sus activos.

- El resto de ratios, basadas en resultados o en variables fondo de la liquidez de las empresas, muestra valores preocupantes. Estos resultados eran de esperar y confirman los negativos efectos de la crisis en las cuentas de pérdidas y ganancias y en la liquidez de las empresas españolas.

Por otro lado, el cuadro 2 presenta el indicador Z-score para cada uno de los sectores de actividad objeto de estudio, distinguiendo también el valor que alcanzan cada uno de sus componentes ponderados.

TABLA 2. Z-SCORE (ALTMAN, 1968) POR SECTORES DE ACTIVIDAD

\begin{tabular}{|c|c|c|c|c|c|c|}
\hline & $Z^{\prime}$ 15 & $\begin{array}{l}1,2 *(\mathrm{FM} / \\
\mathrm{AT})\end{array}$ & $\begin{array}{l}\text { 1,4*(Rdo. no } \\
\text { distr./AT) }\end{array}$ & $\begin{array}{l}3,3^{*}(\mathrm{BAIT} / \\
\mathbf{A T})\end{array}$ & $\begin{array}{l}\text { 0,6*(Capitaliz./ } \\
\text { PT) }\end{array}$ & $\begin{array}{l}\text { 1*(C.N./ } \\
\text { AT) }\end{array}$ \\
\hline $\begin{array}{l}\text { Sector Materiales } \\
\text { Básicos e Industria }\end{array}$ & 12,34 & 0,04 & 0,01 & 0,11 & 11,81 & 0,38 \\
\hline $\begin{array}{l}\text { Sector Servicios de } \\
\text { Consumo }\end{array}$ & 1,44 & $-0,10$ & $-0,04$ & 0,00 & 1,18 & 0,41 \\
\hline $\begin{array}{l}\text { Sector Bienes de } \\
\text { Consumo }\end{array}$ & 10,07 & 0,13 & 0,11 & 0,12 & 9,22 & 0,48 \\
\hline $\begin{array}{l}\text { Sector Tecnología y } \\
\text { Telecomunicaciones }\end{array}$ & 1,37 & $-0,06$ & $-0,06$ & $-0,01$ & 1,08 & 0,42 \\
\hline Sector Inmobiliario & 24,41 & 0,13 & 0,09 & 0,17 & 23,78 & 0,24 \\
\hline $\begin{array}{l}\text { Sector Petróleo y } \\
\text { Energía }\end{array}$ & 184,71 & $-0,06$ & 0,01 & 0,14 & 183,84 & 0,79 \\
\hline
\end{tabular}


Dicho cuadro muestra grandes diferencias entre sectores y confirma la preocupante situación en base a los valores contables. En este sentido, pueden observarse valores negativos para ratios que incluyen variables referentes a resultados o al fondo de maniobra. Por lo tanto, todos los sectores muestran una situación alarmante en términos de liquidez y de resultados. Los valores de mercado vuelven a aparecer como determinantes del resultado del indicador Z-score. En el análisis sectorial destacan los valores mostrados para el sector del petróleo y energía. En este sector el indicador Z-score es especialmente positivo debido a la alta capitalización de las empresas que lo integran. Cabe destacar los valores de Solaria Energía y Medio Ambiente $(Z=686,09)$ e Iberdrola $(Z=593,64)$. Los resultados de dicho sector pueden verse positivamente afectados por la apuesta de muchas grandes empresas eléctricas por las energías renovables. Algunas de estas compañías han llegado a ser referentes mundiales en este campo, lo que ha repercutido favorablemente en su capitalización bursátil. Los sectores que tienen valores de mercado más bajos, servicios de consumo y tecnología y comunicaciones, tienen una alta probabilidad de quiebra, y manifiestan una situación muy delicada.

\subsection{Análisis de la probabilidad de quiebra en base al modelo de Amat (1990)}

El cuadro 3 muestra la información relativa a la probabilidad de quiebra en base al modelo adaptado a las empresas españolas (Amat, 1990). El valor medio del indicador propuesto por Amat (1990) es de 55,55, muy por encima de 0, lo que sugiere una baja probabilidad de quiebra. Sin embargo, la dispersión vuelve a ser significativa, y el valor mediano del mismo, aún mostrando una baja probabilidad de quiebra, se sitúa en 2,93.

En promedio todos los componentes del ratio son positivos destacando al alza el relativo a la liquidez $(45,5)$, que marca la tendencia de este indicador, si bien es cierto que la dispersión de nuevo es muy relevante, aunque el valor mediano se sitúa en 1,55 indicando que al menos la mitad de las empresas analizadas presenta una buena estructura financiera. Es también significativo el peso que tiene el ratio relativo a la independencia financiera. Al contrario de lo observado para el indicador Z-score propuesto por Altman (1968), donde la capitalización juega un papel crucial en los resultados obtenidos, los ratios que en mayor medida condicionan los valores del indicador propuesto por Amat (1990) son aquellos relacionados con la estructura de la deuda. Por otro lado, en cuanto a los beneficios, puede observarse que, como era de esperar, fueron muy reducidos. Los datos apuntan a que al menos el 25 por cien de las empresas obtuvo un resultado negativo en 2015. Esto conduce a valores muy bajos e incluso negativos para los ratios relacionados con la rentabilidad, tanto de activos como de capitales propios.

CUADRO 3. VALORES DESCRIPTIVOS DEL INDICADOR ADAPTADO A LAS EMPRESAS ESPAÑOLAS (AMAT, 1990)

\begin{tabular}{|c|c|c|c|c|c|}
\hline & Media & \begin{tabular}{|c|} 
Desviación \\
estándar
\end{tabular} & $\begin{array}{c}\text { Percentil } \\
25 \\
\end{array}$ & Mediana & \begin{tabular}{|c|} 
Percentil \\
75 \\
\end{tabular} \\
\hline Z empresas españolas & 55,55 & 451,61 & 0,05 & 2,93 & 10,19 \\
\hline $1,35 *(\mathrm{AC} / \mathrm{PC})$ & 45,35 & 403,68 & 0,92 & 1,55 & 2,06 \\
\hline 6,5*(FONDOS PROPIOS/PASIVO) & 13,96 & 49,41 & 2,38 & 4,96 & 10,56 \\
\hline $7 *\left(B^{\circ} \mathrm{NETO} / \mathrm{ACTIVO}\right)$ & 0,25 & 1,01 & $-0,06$ & 0,20 & 0,56 \\
\hline $\begin{array}{l}5^{*}\left(\mathrm{~B}^{\circ} \text { NETO/ CAPITALES }\right. \\
\text { PROPIOS })\end{array}$ & 0,19 & 3,60 & $-0,07$ & 0,29 & 0,86 \\
\hline
\end{tabular}


El cuadro 4 presenta el indicador $\mathrm{Z}$ adaptado a las empresas españolas para cada uno de los sectores de actividad objeto de estudio, distinguiendo además el valor de sus componentes ponderados. Se observan importantes diferencias entre los sectores analizados destacando el sector de petróleo y energía como el menos afectado por el riesgo de quiebra, dada la alta liquidez de las empresas que lo integran. El único sector perjudicado es el de tecnología y comunicaciones debido a que además de obtener un resultado promedio negativo en el año objeto de estudio, su situación es aún más alarmante, ya que las empresas que lo componen no son capaces de hacer frente a la devolución de sus obligaciones a corto plazo. No obstante, a pesar de las diferencias sectoriales, cabe destacar que todos los sectores tuvieron problemas para generar beneficios, obteniendo rentabilidades muy bajas o incluso negativas.

De la comparación de los resultados sectoriales arrojados por ambos modelos pueden extraerse determinadas conclusiones. En primer lugar, a pesar de las diferencias entre los valores obtenidos, a grandes rasgos ambos indicadores coinciden en la clasificación de la mayoría de los sectores en función de su probabilidad de quiebra. Sin embargo, existen algunos sectores donde las divergencias son significativas. Así, ambos modelos difieren en la calificación otorgada al sector de servicios de consumo, que según el modelo de Altman (Altman, 1968) estaría afectado por un alto riesgo de quiebra mientras que no ocurre lo mismo con la adaptación del modelo a las empresas españolas (Amat, 1990).

\section{CUADRO 4. INDICADOR ADAPTADO A LAS EMPRESAS ESPAÑOLAS (AMAT, 1990) POR SECTORES DE ACTIVIDAD}

\begin{tabular}{|c|c|c|c|c|c|}
\hline & $\begin{array}{l}Z \text { empresas } \\
\text { españolas }\end{array}$ & $\begin{array}{c}1,35^{*} \\
\text { (AC/PC) }\end{array}$ & \begin{tabular}{|c|}
$6,5 *(F O N D O S$ \\
PROPIOS/ \\
PASIVO) \\
\end{tabular} & $\begin{array}{c}7^{*}\left(\mathbf{B}^{\circ} \text { NETO/ }\right. \\
\text { ACTIVO) } \\
\end{array}$ & $\begin{array}{l}5^{*}\left(\mathrm{~B}^{\circ} \mathrm{NETO} /\right. \\
\text { CAPITALES } \\
\text { PROPIOS) }\end{array}$ \\
\hline Empresas IGBM & 55,55 & 45,35 & 13,96 & 0,25 & 0,19 \\
\hline $\begin{array}{l}\text { Sector Materiales } \\
\text { Básicos e Industria }\end{array}$ & 5,68 & 1,91 & 7,97 & 0,15 & $-0,14$ \\
\hline $\begin{array}{l}\text { Sector Servicios de } \\
\text { Consumo }\end{array}$ & 3,06 & 1,63 & 6,13 & $-0,08$ & $-0,42$ \\
\hline $\begin{array}{l}\text { Sector Bienes de } \\
\text { Consumo }\end{array}$ & 11,28 & 2,29 & 12,00 & 0,65 & 0,54 \\
\hline $\begin{array}{l}\text { Sector Tecnología y } \\
\text { Telecomunicaciones }\end{array}$ & $-2,25$ & 0,93 & 2,46 & $-0,26$ & $-1,19$ \\
\hline Sector Inmobiliario & 12,27 & 3,69 & 11,75 & 0,48 & 0,55 \\
\hline $\begin{array}{l}\text { Sector Petróleo y } \\
\text { Energía }\end{array}$ & 608,38 & 539,03 & 71,45 & 0,05 & 2,05 \\
\hline
\end{tabular}

Fuente: Elaboración propia.

Por otro lado, el cuadro 5 muestra la comparativa entre el número de empresas que se encuentran en zonas de alto o bajo riesgo de quiebra y aquellas que están en zona gris según los resultados que ofrecen los dos modelos aplicados en este trabajo. 


\section{CUADRO 5. COMPARACIÓN ENTRE EL Z-SCORE DE ALTMAN (1968) Y EL INDICADOR ADAPTADO A LAS ESPAÑOLAS (AMAT, 1990)}

\begin{tabular}{|l|l|l|}
\cline { 2 - 3 } \multicolumn{1}{c|}{} & Z-score Altman & Z-empresas españolas \\
\hline Alta probabilidad de quiebra & 53 & 21 \\
\hline Baja probabilidad de quiebra & 25 & 66 \\
\hline Zona gris & 9 & - \\
\hline Total & 87 & 87 \\
\hline
\end{tabular}

Fuente: Elaboración propia.

Como hemos mencionado anteriormente, ambos modelos muestran coincidencias respecto al análisis de valores agregados. El modelo adaptado a las empresas españolas ofrece resultados que, en promedio, van en la misma línea en lo que al promedio de Z-score de Altman (1968) respecta. Sin embargo, se observan diferencias importantes al realizar una comparación más detallada entre los valores obtenidos por ambos modelos. En particular, la tabla 5 indica que según el modelo de Altman (Altman, 1968) las empresas con alta probabilidad de quiebra son mayores en número que las empresas sanas y que existen un 10 por cien de empresas para las que no está muy clara su exposición al riesgo de quiebra. No obstante, el modelo adaptado a las empresas españolas (Amat, 1990) arroja unos resultados totalmente diferentes en términos de número de empresas, predominando aquellas que ostentan una baja probabilidad de quiebra frente a las que presentan un mayor riesgo de entrar en dificultades financieras.

En base a estos resultados pueden extraerse interesantes conclusiones:

- A pesar de que el indicador de Amat (1990) no incluye valores de mercado, y se base principalmente en valores contables, existen más empresas en situación de baja probabilidad de quiebra que aplicando el indicador de Altman (1968). Las variables consideradas por Amat (1990) y su ponderación suponen por lo tanto un cambio drástico en los resultados obtenidos ya que, en base a valores exclusivamente contables, el modelo propuesto adaptado al contexto español presenta resultados muy diferentes, estando la mayoría de las empresas en una situación favorable.

- Un análisis de la situación en 2018 pone de manifiesto que el modelo de Altman (1968) ha tenido una menor capacidad predictiva. Los resultados sugerían que 53 empresas estaban en alta probabilidad de quiebra y sin embargo apenas algunas de ellas han entrado en problemas financieros graves que desemboquen en concurso de acreedores, causas de disolución, salida de bolsa, etc.

- El modelo de Amat (1990) se muestra a posteriori como más fiable en el contexto español a luz de lo comentado anteriormente. No obstante, atendiendo al número de empresas quebradas o que han sufrido problemas importantes en cuanto a su continuidad, los resultados sugieren que el indicador puede ser mejorable.

- Posibles implicaciones de las divergencias mostradas entre los resultados y la situación real podrían ser la necesidad de revisar ciertas variables a incluir después de varias décadas de cambios económicos, de actualizar ponderaciones, o de realizar adaptaciones para periodos marcados por coyunturas específicas de crisis. 


\subsection{Análisis de la relación entre la probabilidad de quiebra y la calidad de la información voluntaria}

El último objetivo de este trabajo es analizar la posible relación existente entre la probabilidad de quiebra de las empresas y la calidad de la información voluntaria que publican. Los datos descriptivos sobre la calidad de la información voluntaria de las empresas muestran una gran variabilidad a pesar de ser empresas cotizadas. Así, para el año estudiado la empresa con mayor puntuación es Gas Natural, que recibe 86,4 puntos, mientras que la empresa con información voluntaria de menos calidad es Dogi, a la que se lo otorgan 3,8 puntos, siendo el promedio de las empresas de la muestra de 43,2 puntos.

En base a los valores del indicador Z-score de Altman (1968), que es referente en la literatura previa a nivel internacional, se realiza un análisis de diferencias de medias y medianas entre las empresas que se encuentran en zonas de baja probabilidad de quiebra y las que están en zonas de alta probabilidad de quiebra incluyendo la zona gris. Las empresas situadas en la zona gris han sido consideradas comúnmente como empresas con dificultades financieras (Shahwan, 2015). Adicionalmente, se lleva a cabo un análisis basado en el estadístico F-ANOVA para contrastar si las diferencias entre los grupos de empresas en relación a la calidad de la información son significativas. Los resultados se presentan en el cuadro 6.

\section{CUADRO 6. DIFERENCIAS DE MEDIA Y MEDIANA EN LA CALIDAD DE LA INFORMACIÓN VOLUNTARIA}

\begin{tabular}{|l|l|l|l|l|c|}
\cline { 2 - 6 } \multicolumn{1}{c|}{} & \multicolumn{2}{c|}{$\begin{array}{c}\text { Emp. alta prob. quiebra } \\
\text { + zona gris }\end{array}$} & \multicolumn{2}{c|}{ Emp. baja prob. quiebra } & Análisis estadístico \\
\cline { 2 - 6 } \multicolumn{1}{c|}{} & Media & Mediana & Media & Mediana & F-ANOVA \\
\hline $\begin{array}{l}\text { Calidad de la } \\
\text { información } \\
\text { voluntaria }\end{array}$ & 44,68 & 49,1 & 43,01 & 46,15 & $\begin{array}{c}46,15^{* * *} \\
(0,005)\end{array}$ \\
\hline
\end{tabular}

Fuente: Elaboración propia.

Los asteriscos representan el nivel de significatividad: ${ }^{*} p<0.10 ; * * p<0.05$; ${ }^{* * *} p<0.01$

En primer lugar, atendiendo a los valores de la media y la mediana, los resultados ponen de manifiesto que las empresas con mayor riesgo de quiebra llevan a cabo prácticas de divulgación de información voluntaria de más calidad. Adicionalmente, el estadístico FANOVA es significativo al 99 por cien, lo que confirma que las diferencias existentes en los valores medios relativos a la calidad de la información entre ambos grupos de empresas son significativas. La divulgación de la información voluntaria es una de las decisiones empresariales más importantes a la que deben hacer frente todas las empresas debido a las consecuencias que ello supone, ventajas e inconvenientes derivadas de la misma. El hecho de que las empresas que se encuentren en peor situación económica-financiera publiquen información de mayor calidad puede venir explicado por argumentos teóricos en línea con la teoría de la agencia. Estas empresas tienen un mayor nivel de endeudamiento, y por lo tanto mayores asimetrías informativas y conflictos de agencia. Es de esperar por tanto que estas empresas estén sujetas a una presión más elevada en relación con la información que publican. Asimismo, las prácticas de divulgación de información voluntaria son un 
mecanismo clave para reducir dichos conflictos de agencia y mejorar la confianza de los diferentes grupos de interés.

El gráfico 1 muestra cómo la calidad de la información divulgada por las empresas que se encuentran en zonas de alta probabilidad de quiebra es sistemáticamente superior a la de las empresas con baja probabilidad de quiebra.

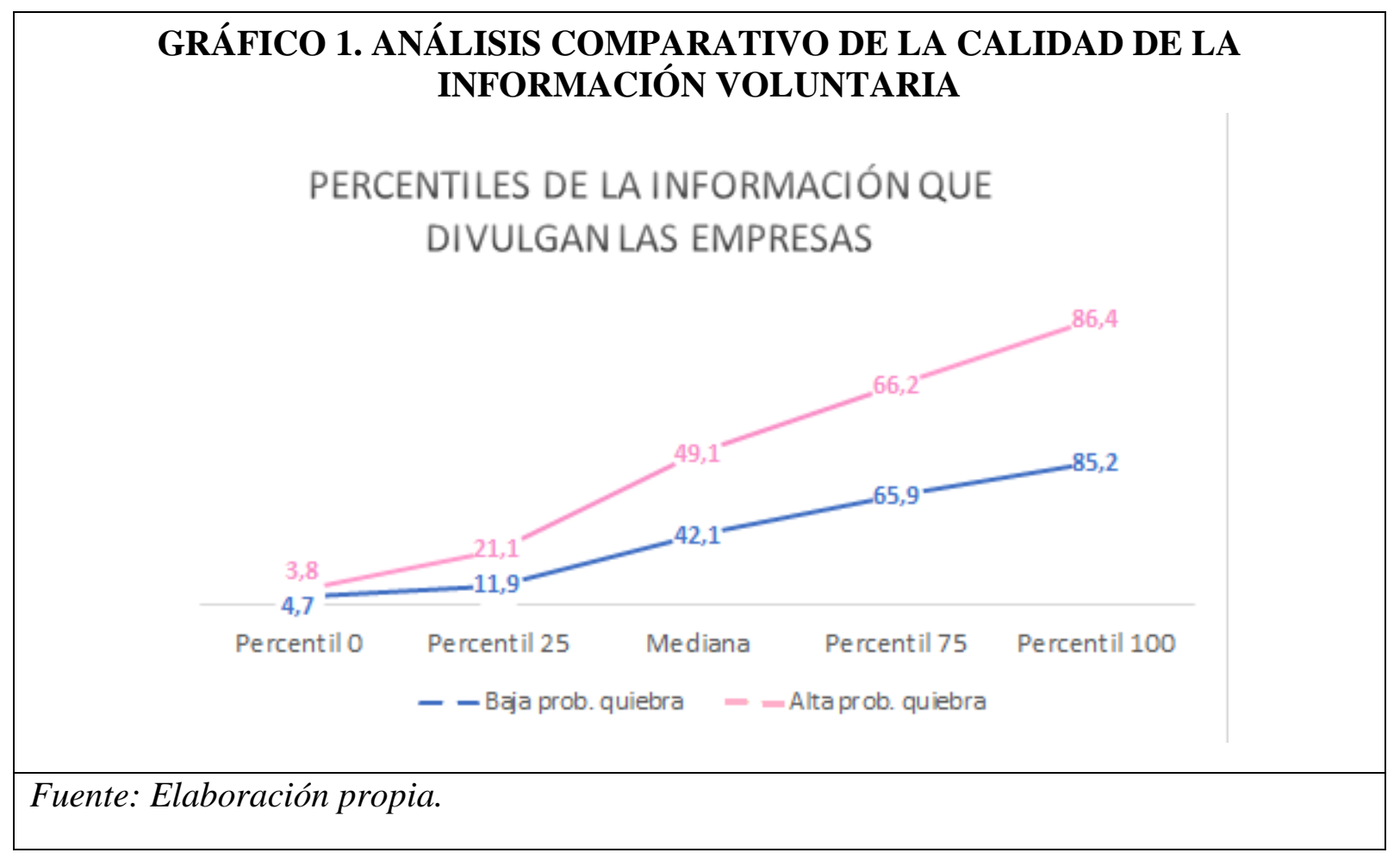

\section{CONCLUSIONES}

Este trabajo pretende dar a conocer la situación respecto a la probabilidad de quiebra de las empresas cotizadas españolas en un momento marcado por una fuerte crisis económica y financiera. Los resultados principales destacan que el indicador Z-score de Altman (1968) está muy condicionado por los valores de mercado, en concreto por la capitalización bursátil. La mayoría de las empresas se encuentran en una situación de alta probabilidad de quiebra, y concretamente la capitalización es la variable que lleva a otras muchas a estar en una situación de baja probabilidad de quiebra. Adicionalmente, este indicador parece no tener suficiente capacidad predictiva en el contexto analizado, en vista de la evolución a posteriori de las empresas estudiadas. Por otra parte, se observan diferencias importantes entre los valores obtenidos por el citado indicador y los procedentes del indicador propuesto por Amat (1990) para el contexto español. Este último indicador parece tener más poder predictivo, aunque los resultados sugieren que existe un margen de mejora, y por lo tanto la conveniencia de adaptar los indicadores de este tipo a ciertos contextos o situaciones coyunturales. Asimismo, la interpretación de los indicadores de probabilidad de quiebra en general debería acompañarse de un análisis cuantitativo y cualitativo adicional de la empresa en cuestión.

Finalmente, se ha realizado un análisis para examinar la relación existente entre la probabilidad de quiebra de las empresas y la calidad de la información voluntaria que 
divulgan. Los resultados corroboran que las empresas en peor situación financiera tienden a tener mejores prácticas de divulgación de información, lo que puede venir explicado por las mayores presiones que tienen y la necesidad de reducir los conflictos de interés con sus diferentes stakeholders.

Este trabajo presenta implicaciones tanto académicas como prácticas. A nivel académico, este estudio se centra en evaluar la capacidad predictiva de los indicadores de probabilidad de quiebra en un escenario particular, y los resultados pueden motivar futuras investigaciones relativas al diseño de indicadores adaptados a contextos específicos. Asimismo, el estudio desarrollado supone un avance en la literatura, mostrando nueva evidencia sobre los determinantes de la calidad de la información voluntaria y, en otro sentido, consecuencias de la probabilidad de quiebra. Por último, los resultados obtenidos pueden ser relevantes tanto para reguladores como para empresas.

\section{BIBLIOGRAFÍA}

Al-Hadi, A., Chatterjee, B., Yaftian, A., Taylor, G. y Hasan, M. M. (2017): "Corporate social responsibility performance, financial distress and firm life cycle: evidence from Australia”, Accounting and Finance.

Altman E.I. (1983): "Corporate Financial Distress and Bankruptcy: A complete Guide to predicting and avoiding distress and profiting from bankruptcy", https://doi.org/10.111/acfi.12277.

Altman E.I., Hartzell J. y Peck M. (1995): Emerging Markets Corporate Bonds: A Scoring System, Salomon Brothers Inc, New York.

Altman, E. I. (1968): "Financial ratios, discriminant analysis and the prediction of corporate bankruptcy”, The journal of finance, vol. 23, n 4, pp. 589-609.

Altman, E.I. y Hotchkiss, E. (2006): Corporate Financial Distress \& Bankruptcy. 3rd edition. J. Wiley \& Sons. Hoboken. New Jersey.

Altman, E.I., Danovi, A. y Falini, A. (2015): “Z-Score Models' Application to Italian Companies Subject to Extraordinary Administration”, Journal of Applied Finance (Formerly Financial Practice and Education), vol 23, n ${ }^{\circ} 1$.

Amat, O. (1990): Predicción del éxito o del fracaso de una empresa en base a variables cualitativas y cuantitativas (Tesis doctoral), Universidad Autónoma de Barcelona

Beaver, W. (1966): “Financial Ratios as Predictors of Failure”, Journal of Accounting Research, $\mathrm{n}^{\circ}$ 4, pp. 71-111.

Berrío, D. y Cabeza, L. (2003): "Verificación y adaptación del modelo de Altman a la superintendencia de sociedades de Colombia”, Pensamiento y Gestión, n 15, pp. 2651

Cabrera, J. (2008): “La transparencia es clave para el futuro de nuestras organizaciones”. Recuperado el 27 de marzo de 2017 de https://blog.cabreramc.com/2008/10/09/latransparencia-es-clave-para-el-futuro-de-nuestras-organizaciones/

Celli, M. (2015): “Can Z-score model predict listed companies’ failures in Italy? An empirical test”, International Journal of Business and Management, vol. 10, n 3, pp. 57-66.

Charitou, A., Lambertides, N. y Trigeorgis, L. (2011): "Distress risk, growth and earnings quality”, ABACUS no 47, pp. 158-181. 
Chen, Y., Chien, C. y Huang, S. (2010): “An appraisal of financially distressed companies’ earnings management: evidence from listed companies in China, Pacific”, Accounting Review $\mathrm{n}^{\circ}$ 22, pp. 22-41.

Francis, J., Nanda, D. y Olsson P. (2008): "Voluntary Disclouse, Earnings Quality, and Cost of Capital”, Journal of Accounting Research, 46, n¹, pp. 53-99.

Galindo, R. (2000): Teoría y aplicación del principio de empresa en funcionamiento: estudio empírico (Tesis doctoral), Universidad Autónoma de Madrid.

García-Meca, E., Parra, I., Larrán, M. y Martínez, I. (2005): “The explanatory factors of intellectual capital disclosure to financial analysts", European Accounting Review, vol.14, nº1, pp. 63-94.

Gerantonis, N., Vergos, K. y Christopoulus, A. (2009): "Can Altman Z-score model predict business failures in Greece?”, Research Journal of International Studies, $\mathrm{n}^{\mathrm{0}}$ 12, pp. 2128.

Habib, A., Costa, M. D., Huang, H. J., Bhuiyan, M. B. U. y Sun, L. (2018): "Determinants and consequences of financial distress: review of the empirical literature”, Accounting and Finance, https://doi.org/10.1111/acfi.12400.

Healy, P.M. y Palepu, K.G. (2001): "Information asymmetry, corporate disclosure, and the capital markets: A review of the empirical disclosure literature", Journal of Accounting and Economics $\mathrm{n}^{\circ}$ 31, pp. 405-440.

Holder-Webb, L. y Cohen, J. R. (2007): "The association between disclosure, distress, and failure", Journal of Business Ethics n ${ }^{\circ} 75$, pp. 301-314.

Ibarra, A. (2001): Análisis de las dificultades financieras de las empresas en una economía emergente: las bases de datos y las variables independientes en el sector hotelero de la bolsa mexicana de valores (Tesis doctoral), Universidad Autónoma de Barcelona.

InformeReporta (2016): El Informe Reporta 2016. Recuperado el 28 de marzo de 2017 de http://informereporta.com/acerca-de

Jacoby, G., Li, J. y Liu, M. (2016): "Financial distress, political affiliation and earnings management: the case of politically affiliated private firms”, The European Journal of Finance.

Jensen, M. y Meckling, W. (1976): "Theory of the firm: Managerial behavior, agency cost and ownership structure”, Journal of Financial Economics, n ${ }^{\circ}$ 3, pp. 305-360.

Kane, G. D., Velury, U. y Ruf, B. M. (2005): "Employee relations and the likelihood of occurrence of corporate financial distress", Journal of Business Finance and Accounting, $\mathrm{n}^{\circ}$ 32, pp. 1083-1105.

Lambert, F., Bigler, M., Steffensen, J. P., Hutterli, M. A. y Fischer, H. (2012): "EDC dust flux $800 \mathrm{kyr}$ (25 yr mean)”, PANGAEA.

Lee, J. E., Glasscock, R. y Park, M. S. (2017): "Does the ability of operating cash flows to measure firm performance improve during periods of financial distress?”, Accounting Horizons, $n^{\circ} 31$, pp. 23-35.

Ley 19/2013, de 9 de diciembre, de transparencia, acceso a la información pública y buen gobierno. Boletín Oficial del Estado, núm. 295, de 10 de diciembre de 2013, pp. 97922 a 97952. Recuperada de https://www.boe.es/buscar/doc.php?id=BOE-A-2013-12887.

Ley 22/2003, 9 de julio, Concursal. Boletín Oficial del Estado, núm. 164, de 10 de julio de 2003. Recuperado de https://www.boe.es/buscar/doc.php?id=BOE-A-2003-13813. 
Llenas, J. (2006): Análisis y plan estratégico, económico y financiero, análisis sistema de costes y estudio proyecto de inversión de una empresa familiar fabricante de equipamiento médico (Trabajo Fin de Master), Universidad de Barcelona.

Manzaneque M., Priego, A. y Merino, E. (2016): “Corporate Governance effect on finalcial distress likelihood: Evidence from Spain”, Revista de Contabilidad- Spanish Accounting Review, vol. 19, $\mathrm{n}^{\mathrm{o}}$ 1, pp. 111-121.

Mohammed, A. A. E. y Kim-Soon, N. (2012). "Using Altman's model and current ratio to assess the financial status of companies quoted in the Malaysian stock exchange", International Journal of Scientific and Research Publications, vol. 2, n 7, pp. 1-11.

Mora, A. (1994): "Los modelos predicción del fracaso empresarial: una aplicación empírica del logit”, Revista Española de Financiación y Contabilidad, vol. XXIV n ${ }^{\circ}$ 78, pp. 203-233

Real Decreto de 22 de agosto de 1885 por el que se publica el Código de Comercio. Ministerio de Gracia y Justicia BOE núm. 289, de 16 de octubre de 1885, referencia: BOE-A-1885-6627. Recuperado de https://www.boe.es/buscar/act.php?id=BOE-A1885-6627.

Richardson, G., Lanis, R. y Taylor, G. (2015): "Financial distress, outside directors and corporate tax aggressiveness spanning the global financial crisis: an empirical analysis”, Journal of Banking and Finance, $n^{\circ}$ 52, pp. 112-129.

Rodríguez, N. y Gómez A. (2016): “Utilización de indicadores para la predicción de insolvencia”, Revista de Contabilidad y Dirección, n 23, pp. 179-195.

Sarkar, S. y Bhattacharjee, T. (2017): "Impact of Voluntary Disclosures on Corporate Brand Equity”, Corporate Reputation Review, vol. 20, n 2, pp. 125-136.

Shahwan, T. M. (2015): "The effects of Corporate Governance on financial performance and financial distress: evidence from Egypt”, Corporate Governance, vol. 15, $\mathrm{n}^{\circ}$ 5, pp. 641-662.

Thomas, S., Wong, J. y Zhang, J. (2011): “Applying Z-score model to distinguish insolvent construction companies in China”, Habitat international, vol. 35, n 4, pp. 599-607.

Tykvová, T. y Borell, M. (2012): "Do private equity owners increase risk of financial and bankruptcy?”, Journal of Corporate Finance n 18 , pp. 138-150.

Udin, S., Arshad, M. y Javid, A.J. (2017): “The effects of ownership structure on likelihood of financial distress: an empirical evidence", Corporate Governance: The International Journal of Business in Society, vol. 17, $\mathrm{n}^{\circ}$ 4, pp. 589-612.

Valencia, M., Tróchez, J., Vengas J.G. y Restrepo, J.A. (2016): “Modelo para el análisis de la quiebra financiera en pymes agroindustriales antioqueñas", Apuntes del CENES, vol. 35, $\mathrm{n}^{\circ}$ 62, pp. 147-168.

Vargas, J.A. (2013): “Modelos para la prevención de bancarrotas empresariales utilizados por el sector empresarial costarricense”, Tec Empresarial, vol. 7, nº 3, pp. 43-49. 